Background Prothrombotic risk factors (PRF) are suggested to be involved in the pathogenesis of PAIS. However, most of published studies are retrospective, they vary in the PRF tested and parents are not usually investigated.

Objective To determine the impact of parental and infant thrombophilia on neonatal cases diagnosed of PAIS in a prospective case-control study.

Methods Factor V (G1691A mutation), prothrombin G20210A variant, MTHFR C677T genotype, antithrombin, protein C, protein S, lipoprotein (a), homocystein (Hcy) and anticardiolipin antibodies were investigated in 45 infant-parent pairs with PAIS and in 85 controls. Blood samples were drawn within the first week of life.

Results All thrombophilic factors investigated were similar or even less frequent among patients with PAIS and their parents compared to controls. The most frequent PFRs were Hcy $>11$ and MTHFR homozygosity in cases $(7.5 \%$ and $5.3 \%$, respectively) and in controls (24.4\% and $13.1 \%$, respectively). Thirteen neonates diagnosed of PAIS (28.9\%) had at least 1 PFR, compared to 39 subjects $(44.3 \%)$ in the control group (OR/95\% CI, $0.51 / 0.23$ to 1.10$)$ ( $p<0.001)$. Twenty three mothers of infants with PAIS (51.1\%) were positive for thrombophilia markers, compared to $49(55.7 \%)$ controls $(p=.617)$. In 8 mother-infant pairs $(17.8 \%)$, at least 1 PFR could be identified for either mother or infant, compared to 19 controls $(21.6 \%)$. Fifteen neonates with stroke $(33.3 \%)$ had at least one PFR compared to the 57 subjects $(64.8 \%)$ in the control group $(\mathrm{p}<$ 0.001).

Conclusion Our data do not support that PFR play a major role in PAIS.

\section{PO-0407 PERINATAL FACTORS AND PERINATAL ARTERIAL ISCHAEMIC STROKE (PAIS): A PROSPECTIVE CASE-CONTROL STUDY}

${ }^{1} \mathrm{~A}$ Arnaez, ${ }^{2} \mathrm{G}$ Arca-Diaz, ${ }^{2} \mathrm{~A}$ Martin-Ancel, ${ }^{2} \mathrm{~T}$ Agut, ${ }^{2} \mathrm{M}$ Camprubí, ${ }^{2} \mathrm{~A}$ García-Álix. ${ }^{1}$ Neonatology, University Hospital of Burgos, Burgos, Spain; ${ }^{2}$ Neonatology, Agrupacio Sanitaria Hospital Sant Joan de Déu-Hospital Clinic, Barcelona, Spain

\subsection{6/archdischild-2014-307384.1053}

Background Perinatal factors (PF) have been implicated in the pathogenesis of PAIS. Hypoxia has been suggested to be involved as a potential cause of AIS although prospective case-control studies are still warranted.

Objective To determine the impact of PF on neonatal cases diagnosed of PAIS in a prospective case-control study.

Methods 45 neonates were diagnosed of PAIS within four weeks of life and 85 controls were investigated for the following PF: retarded intrauterine growth restriction, sentinel event, epidural and general anaesthesia, presentation at delivery, type of delivery (vaginal, instrumental, emergency caesarean section), abnormal cardiotocographic monitoring, Apgar score at 1 and $5 \mathrm{~min}$, arterial umbilical cord $\mathrm{pH}<7.0$ and $<7.20$, and need of advanced resuscitation.

Results The univariate analysis indicated the following associations with PAIS: emergency caesarean section $(48.9 \%$ vs $14.8 \%$; $\mathrm{p}<0.001)$; Apgar score at $1 \mathrm{~min}<7(22.2 \%$ vs $4.6 \% ; \mathrm{p}=$ $0.005)$ and $<5(15.6 \%$ vs $1.1 \% ; \mathrm{p}=0.002)$; arterial cord $\mathrm{pH}$, mean \pm SD $7.19 \pm 0.12(\mathrm{CI} 95 \% 7.15,7.23)$ in infants with PAIS compared to $7.25 \pm 0.1(7.23,7.27)$ in controls $(\mathrm{p}=$ $.005)$; arterial cord $\mathrm{pH}<7.20$ (45.2\% vs $17.8 \%$; $\mathrm{p}=0.006)$.

A multivariate analysis did not show any independent factor associated to PAIS, except for arterial cord $\mathrm{pH}<7.20$ (OR 2.89,
CI95\% 1.01, 8.31). Emergency caesarean section and Apgar score $<5$ at $1 \mathrm{~min}$, showed a tendency to be associated with PAIS but without statistical significance: OR 2.61 (95\% CI 0.66, 10.2) and 10.02 (CI95\% 0.46, 215.8).

Conclusion Our data indicate that PF do not appear to play a major role in PAIS.

\section{PO-0408 CAUSES AND OUTCOME OF NEONATAL CONVULSIONS}

F Kamoun, M Hsairi, L Sfaihi, S Ben Ameur, I Maaloul, TH Kamoun, M Hachicha. Pediatrics Department, Hedi Chaker Hospital, Sfax, Tunisia

\subsection{6/archdischild-2014-307384.1054}

Background and aims Convulsions occur more frequently in the neonatal period than at any other time.

Aims Study the clinical, etiological, therapeutic aspects and the outcome of neonatal convulsions.

Methods We report a retrospective analysis of 51 cases of neonatal convulsions between 2009 and 2013.

Results The sex-ratio was 1.1. Prematurity was found in 8 patients. The Apgar score was lower or equal to 7 in the first minute in 16 cases. The average age of the first convulsion was 9 days. The motive of admission was convulsion in 35 cases (66\%). It was a convulsive status epilepticus in 4 cases. The neurological examination was pathological in 27 cases. A metabolic balance sheet, lumbar puncture were realised in every case, cranial ultrasound scan (34 cases), cerebral CT scan (19 cases), electroencephalography (29 cases) and MRI (16 cases). Causes of neonatal convulsions were: hypoxic-ischaemic encephalopathy (13 cases), hypoglycemia (7 cases), hyponatraemia (6 cases), hypocalcemia (4 cases), pyridoxine dependency (1 case), meningitis (5 cases), hypertension (1 case), ischaemic stroke (1 case), cerebral haemorrhage (3 cases), anomaly of the urea cycle (1 case), West syndrome (2 cases) and idiopathic neonatal convulsions ( 7 cases). The treatment was etiological in 23 cases. The outcome was favourable in 37 cases, 4 patients died and variable sequelae were found in 10 cases.

Conclusion This study emphasises the variability of aetiology of neonatal convulsions and the necessity of the etiological diagnosis on which will depend the prognosis.

\section{PO-0409 WITHDRAWN}

\section{PO-0410 PROPYLENE GLYCOL (PG) DETECTION BY BRAIN MAGNETIC SPECTROSCOPY (MRS) IN INFANTS WITH NEONATAL ENCEPHALOPATHY}

${ }^{1} \mathrm{~K}$ Povazai, ${ }^{2} \mathrm{R}$ Johnstone, ${ }^{2} \mathrm{~N}$ Byrne, ${ }^{2} \mathrm{~J}$ Ashmore, ${ }^{3} \mathrm{D}$ Azzopardi, ${ }^{4} \mathrm{MM}$ U-King-Im, ${ }^{4} \mathrm{~A}$ Siddiqui, ${ }^{1} \mathrm{AB}$ Kapetanakis, ${ }^{2} \mathrm{G}$ Charles-Edwards. 'Neonatal Medicine, Evelina London Children's Hospital, London, UK; ${ }^{2}$ Medical Physics, King's College London, London, UK; ${ }^{3}$ Neonatal Medicine, King's College London, London, UK; ${ }^{4}$ Neuroradiology, Guy's and St Thomas NHS Foundation Trust, London, UK

\subsection{6/archdischild-2014-307384.1055}

Background PG is a commonly used excipient contained in several medications to control neonatal seizures (Phenobarbitone (PhB) (79\%) Phenytoin (Ph) (40\%) and Clonazepam). On MRS the appearance of a doublet at $1.1 \mathrm{ppm}$ in some spectra is attributed to PG. The lower safe level of short term exposure to PG 